\title{
GENERIC ISOTOPIES OF SPACE CURVES
}

\author{
by J. W. BRUCE and P. J. GIBLIN
}

(Received 25 March, 1985)

For a single space curve (that is, a smooth curve embedded in $\mathbb{R}^{3}$ ) much geometrical information is contained in the dual and the focal set of the curve. These are both (singular) surfaces in $\mathbb{R}^{3}$, the dual being a model of the set of all tangent planes to the curve, and the focal set being the locus of centres of spheres having at least 3-point contact with the curve. The local structures of the dual and the focal set are (for a generic curve) determined by viewing them as (respectively) the discriminant of a family derived from the height functions on the curve, and the bifurcation set of the family of distance-squared functions on the curve. For details of this see for example [6, pp. 123-8].

In this paper we consider instead a smooth one-parameter family of space curves, which we refer to as an "isotopy" since for our transversality result of $\$ 6$ we do need to assume that it comes from an ambient isotopy of $\mathbb{R}^{3}$. We determine all the generic transitions which occur on the dual and the focal set during this isotopy. Thus, for example, at isolated moments of the isotopy there may be a point of zero curvature on the curve, in which case every tangent plane at such a point is an osculating plane and the dual acquires an extra cuspidal edge. Where this appears from (and goes to) can be deduced from the geometry of the family of duals as described in $\$ 2$ below.

The work in this paper uses essentially the same methods as a previous study of the plane curve case by one of us [4], and so relies much on the results of Arnold [1]; see also [2].

In $\$ 1$ and $\$ 3$ we determine the conditions under which an isotopy gives rise to the local generic changes in dual and focal set that can be recognised by Arnold's results. In $\$ 6$ a transversality theorem is proved which shows that the set of such isotopies is open and dense. Meanwhile in $\$ 2$ and $\$ 4$ we give explicit examples of some of the more interesting transitions in order to make the results easier to understand, and in $\$ 5$ we consider some of the "multi-local" questions which arise. Thus, for example, the dual acquires self-intersections because a single plane can be tangent at two (widely separated) points of the curve, and we show how to determine the conditions for all the stable transitions which occur on the dual near these self-intersections. The results here are very much what one would expect, and we do not dwell on them at length.

Throughout this paper smooth means infinitely differentiable, and all curves and maps considered will be smooth.

Since the authors submitted this paper it has come to their attention that there is some overlap of results discussed here with those in a paper of Shcherbak [8]. In particular Shcherbak discusses fronts of space curves: these coincide with our duals discussed in $\$ 1$ below.

Glasgow Math. J. 29 (1987) 41-63. 
1. Height functions. Let $C$ be a circle, $\phi: C \rightarrow \mathbb{R}^{3}$ an embedding and $S^{2}$ the unit sphere in $\mathbb{R}^{3}$ giving all directions in space. We shall use the families of functions on $C$

$$
h: C \times S^{2} \rightarrow \mathbb{R}
$$

and

$$
f: C \times S^{2} \times \mathbb{B} \rightarrow \mathbb{R}
$$

given by

$$
h(t, u)=\phi(t) \cdot u
$$

and

$$
f(t, u, w)=\phi(t) \cdot u-w
$$

The discriminant of $f$,

$$
\mathscr{D}(f)=\left\{(u, w): f(t, u, w)=\frac{\partial f}{\partial t}(t, u, w)=0 \text { for some } t\right\}
$$

can be regarded as the affine dual of $\phi(C)$, since $(u, w)$ in $\mathscr{D}(f)$ determines the tangent plane $x \cdot u=w$ to $\phi(C)$. (Compare [6, p. 126]. Strictly we should factor by the equivalence relation $(u, w) \sim(-u,-w)$ but for examining the dual locally, or multilocally, $\mathscr{D}(f)$ will serve.) Also, the smooth 2-manifold

$$
\sigma=\left\{(t, v): \frac{\partial h}{\partial t}(t, v)=0\right\}
$$

is the unit normal bundle to $\phi(C)$ (a circle bundle over $C$ ), and the natural projection $p: \sigma \rightarrow S^{2}$ is the Gauss map of $\phi(C)$. (Again we could, but don't, factor $S^{2}$ by $v \sim-v$.)

The set of critical values of the Gauss map can be described in various ways, such as:

(i) the bifurcation set of the family $h$;

(ii) the discriminant set $\mathscr{D}(\partial h / \partial t)$ of the family $\partial h / \partial t$;

(iii) the projection to $S^{2}$ of the cusp edges on the dual.

Using (ii) we can deduce the local structure of the set of critical values at the same time as deducing that of the dual. This is more significant when we come to a family $\Phi$ of space curves (below), since with a single curve we can even say what the Gauss map itself is-see (1.1).

The following result is well known-see [6, p. 127] for most of it. Suppose that $\phi(C)$ has nowhere zero curvature and that, whenever the torsion $\tau$ vanishes its derivative does not. (These both hold for a generic curve $\phi(C)$.) Let $h(-, v)$ have type $A_{k}$ at $t$, and write $w=h(t, v)$.

(1.1) Proposition. With the above hypotheses,

(a) $k=1 \Leftrightarrow v$ is normal to $\phi(C)$ at $\phi(t)$ but not normal to the osculating plane there. When $k=1$, the dual is smooth at $(v, w)$ and the Gauss map is a local diffeomorphism at $(t, v)$, critical set empty. 
(b) $k=2 \Leftrightarrow v$ is normal to the osculating plane at $\phi(t)$ but $\tau(t) \neq 0$. When $k=2$, the dual is a cusp edge at $(v, w)$ and the Gauss map has a fold at $(t, v)$, critical set smooth.

(c) $k=3 \Leftrightarrow v$ is normal to the osculating plane, $\tau(t)=0\left(\right.$ so $\left.\tau^{\prime}(t) \neq 0\right)$. When $k=3$, the dual is a swallowtail at $(v, w)$ and the Gauss map has a cusp (Whitney pleat) at $(t, v)$, critical set an ordinary cusp.

Note that the exclusion of $\tau=\tau^{\prime}=0$ makes $k>3$ impossible.

We now turn to a one-parameter family of curves. For most of the calculations below it is enough to assume that we are given a smooth map

$$
\Phi=\left(\Phi_{1}, \Phi_{2}, \Phi_{3}\right): C \times U \rightarrow \mathbb{R}^{3},
$$

where $U$ is an open interval of $\mathbb{R}$ containing $[0,1]$. (Thus initially $U$ can be $(-1,2)$ say, but it may shrink.) This is regarded as a deformation of $C_{0}=\Phi(C \times\{0\})$ to $C_{1}=$ $\Phi(C \times\{1\})$. When proving our transversality theorem in $\$ 6$, however, we do need to make explicit use of an ambient isotopy, that is a smooth map

$$
\Omega: \mathbb{R}^{3} \times U \rightarrow \mathbb{R}^{3}
$$

for which each map $\Omega(-, u)$ is a diffeomorphism. A family $\Phi$ is induced from $\Omega$ and an initial embedded curve $\Phi(-, 0)$ in the obvious way:

$$
\Phi(t, u)=\Omega(\Phi(t, 0), u) .
$$

There are associated big height functions:

$$
\begin{gathered}
H: C \times U \times S^{2} \rightarrow \mathbb{R} \\
F: C \times U \times S^{2} \times \mathbb{R} \rightarrow \mathbb{R}
\end{gathered}
$$

given by

$$
H(t, u, v)=\Phi(t, u) \cdot v
$$

and

$$
F(t, u, v, w)=\Phi(t, u) \cdot v-w .
$$

Thus the "big dual" is the discriminant $\mathscr{D}(F)$ of $F$ and the set of critical values of the "big Gauss map" is the discriminant $\mathscr{D}(\partial H / \partial t)$ of $\partial H / \partial t$. We need to determine when the projections of these discriminants to $U$ are generic functions in Arnold's sense. We shall do this, for convenience, at the values $t=0, u=0 \in U, v=(0,0,1)$. Write

$$
\left.\begin{array}{c}
\Phi(t, 0)=\left(t, \phi_{2}(t), \phi_{3}(t)\right) \\
\frac{\partial \Phi}{\partial u}(t, 0)=\left(\psi_{1}(t), \psi_{2}(t), \psi_{3}(t)\right)
\end{array}\right\}
$$


where

$$
\begin{aligned}
& \phi_{2}(t)=a_{2} t^{2}+a_{3} t^{3}+a_{4} t^{4}+a_{5} t^{5}+\text { h.o.t. } \\
& \phi_{3}(t)=b_{2} t^{2}+b_{3} t^{3}+\text { h.o.t. } \\
& \psi_{1}(t)=c_{0}+c_{1} t+c_{2} t^{2}+\text { h.o.t. } \\
& \psi_{2}(t)=d_{0}+d_{1} t+d_{2} t^{2}+\text { h.o.t. } \\
& \psi_{3}(t)=e_{0}+e_{1} t+e_{2} t^{2}+\text { h.o.t. }
\end{aligned}
$$

The first thing to observe is that for local calculations we can replace $F$ by the germ (also called $F$ )

$$
\begin{gathered}
F: \mathbb{R} \times \mathbb{R}^{2} \times \mathbb{R} \times \mathbb{R},(0,0,0,0) \rightarrow \mathbb{R} \\
(t, v, u, w) \rightarrow v_{1} \Phi_{1}+v_{2} \Phi_{2}+\Phi_{3}-w
\end{gathered}
$$

(That is, $S^{2}$ is replaced by $\left\{\left(v_{1}, v_{2}, 1\right)\right\}$.) Similarly with $H$. It is then clear that

$$
\begin{aligned}
& F(-, 0,0,0) \text { or } H(-, 0,0) \text { has type } A_{k} \text { at } t=0 \text { if and only if } \\
& b_{2}=\ldots=b_{k}=0, b_{k+1} \neq 0 .
\end{aligned}
$$

Next we need the conditions for $F$ to be a versal unfolding of this function at $t=0$, so that the dual $\mathscr{D}(F)$ is locally diffeomorphic to a standard discriminant of $A_{k}$ in $\mathbb{R}^{4}$. These conditions coincide with those for the critical values of the Gauss map $\mathscr{D}(\partial H / \partial t)$ to be a standard discriminant-but of $A_{k-1}$ this time-in $\mathbb{R}^{3}$. The conditions are:

$$
\begin{aligned}
& k=1: \text { no condition } \\
& k=2: \text { no condition } \\
& k=3: a_{2} \neq 0 \text { or } e_{2} \neq 0 \\
& k=4: a_{2} e_{3}-a_{3} e_{2} \neq 0 .
\end{aligned}
$$

Finally we need the additional conditions for the projection $\mathscr{D}(F) \rightarrow U$ to be one of Arnold's generic functions. (Again these coincide with the conditions for $\mathscr{D}(\partial H / \partial t) \rightarrow U$ to be generic.) The conditions are obtained as in [3]; we shall state them here and then give more detail in one case.

Arnold's generic functions [1] have the following form for a standard $(k+n)$ parameter versal unfolding

$$
\begin{gathered}
\mathbb{R} \times \mathbb{R}^{k} \times \mathbb{R}^{n} \rightarrow \mathbb{R} \\
\left(t, \lambda_{1}, \ldots, \lambda_{k}, \tau_{1}, \ldots, \tau_{n}\right) \rightarrow \pm t^{k+1}+\lambda_{1} t^{k-1}+\lambda_{2} t^{k-2}+\ldots+\lambda_{k}
\end{gathered}
$$

of a standard $A_{k}$ singularity $(k \geqslant 1, n \geqslant 0)$, at $t=0$ :

$$
\begin{aligned}
& (\lambda, \tau) \rightarrow \lambda_{1} \pm \tau_{1}^{2} \pm \ldots \pm \tau_{n}^{2}, \quad \text { (signs independent) } \\
& (\lambda, \tau) \rightarrow \tau_{1} .
\end{aligned}
$$


The second we call trivial; the first, for $n>0$, is called a Morse function. For $n=0$ we call it the standard projection.

In the present case (where $k+n=4$ for $F$ and for $\partial H / \partial t$ ) the additional conditions are as follows, where we write $x$ for the curvature of the $u=0$ curve.

$$
\begin{aligned}
& k=1 \text { or } 2 \text { : projection always trivial. } \\
& k=3:\left\{\begin{array}{l}
\text { trivial } \Leftrightarrow a_{2} \neq 0 \Leftrightarrow \kappa(0) \neq 0, \\
\text { Morse }\left(\sim \pm\left(\lambda_{1}+\tau_{1}^{2}\right)\right) \Leftrightarrow a_{2}=0, a_{3} \neq 0 \Leftrightarrow \kappa(0)=0,\left(\kappa^{2}\right)^{\prime \prime}(0) \neq 0 .
\end{array}\right. \\
& k=4: \text { standard } \Leftrightarrow a_{2} \neq 0 \Leftrightarrow \kappa(0) \neq 0 .
\end{aligned}
$$

Note that we have to say $\left(\kappa^{2}\right)^{\prime \prime}(0) \neq 0$ rather than $\kappa^{\prime}(0) \neq 0$ since $\kappa$ is not differentiable when $K=0$. The easiest way to check the connections with curvature is to use the formula $\kappa=\left|\gamma^{\prime} \times \gamma^{\prime \prime}\right| /\left|\gamma^{\prime}\right|^{3}$ for a parametrized curve $\gamma$, remembering that $b_{2}=0$ for $k \geqslant 3$, by (1.4). We also note that there is a unique direction in which the height function has type $A_{\geqslant 2}$ at $t=0$, if and only if $a_{2} \neq 0$ (i.e., $K(0) \neq 0$ ), and that when $a_{2}=0$ there is a unique direction giving $A_{\geqslant 3}$ at $t=0$, if and only if $a_{3} \neq 0$.

Most of the conditions in (1.7) are very easy to check. Triviality requires that the $(k-1)$-jets (with constant) of $\partial F / \partial v_{1}, \partial F / \partial v_{2}, \partial F / \partial w$ span $\mathbb{R}[t] /\left\langle t^{k}\right\rangle$, while for a standard projection the $(k-2)$-jets need to span $\mathbb{R}[t] /\left\langle t^{k-1}\right\rangle$.

The Morse case requires more calculation. Assuming $b_{2}=b_{3}=0, b_{4} \neq 0, a_{2}=0$, $e_{2} \neq 0$ (from (1.4), (1.5) and the assumed non-triviality of the projection), $\mathscr{D}(F)$ is of the form "swallowtail $\times \mathbb{R}$ " and the set of $A_{3}$ points is the "swallowtail edge", corresponding to the $\mathbb{R}$ factor. We need to check (see [3]):

(i) the projection restricted to a section of $\mathscr{D}(F)$ transverse to the swallowtail edge is a generic function on the swallowtail;

(ii) the projection restricted to the swallowtail edge is a Morse function $\mathbb{R} \rightarrow \mathbb{R}$, in the ordinary sense.

We use the map

$$
\theta: \mathbb{R} \times \mathbb{R}^{2} \times \mathbb{R} \times \mathbb{R} \rightarrow \mathbb{R}^{4}
$$

taking $(t, v, u, w)$ to the 3-jet with constant of $F(-, v, u, w)$ at $t$. Then $\theta^{-1}(0)$ is, close to 0 , the swallowtail edge. The Jacobian matrix of $\theta$ at $t=0$ is

$$
\left[\begin{array}{ccccr}
0 & 0 & 0 & e_{0} & -1 \\
0 & 1 & 0 & e_{1} & 0 \\
0 & 0 & 0 & 2 e_{2} & 0 \\
24 b_{4} & 0 & 6 a_{3} & 6 e_{3} & 0
\end{array}\right]
$$

Columns $1,2,4,5$ are linearly independent, so $v_{2}$ is a local coordinate on the swallowtail edge, and $v_{2}=0$ is transverse to the edge. For (i) we only need the 1 -jets of $\partial F / \partial v_{1}$, $\partial F / \partial w$ to span $\mathbb{R}[t] /\left\langle t^{2}\right\rangle$, which is easily checked to be so.

For (ii) we can assume $v_{1}, u, w$ and $c$ are functions of $v_{2}$ on $\theta^{-1}(0)$ and proceed to calculate $u^{\prime \prime}(0)$ : it comes to $3 a_{3}^{2} / 4 b_{4} e_{2}$, so that indeed $u^{\prime \prime}(0) \neq 0 \Leftrightarrow a_{3} \neq 0$. The fact that the 
normal form is $\pm\left(\lambda_{1}+\tau_{1}^{2}\right)$ actually follows geometrically, for the sections of the standard discriminant given by $\lambda_{1}-\tau_{1}^{2}=$ constant are impossible for the dual of a space curve. These sections are drawn in [2, p. 31, Fig. 32(1)] and [1, p. 577, second from right], and some of them have no cusp edge; however, the cusp edge corresponds to osculating planes of the space curve, which always exist. (Equally the critical points of the Gauss map cannot exhibit a "lips" transition.) Naturally this geometrical fact also follows by calculation: in fact using the method of [7] to find normal forms explicitly we find that the sign of $\lambda_{1}$ is that of $b_{4} e_{2}$ and the sign of $\tau_{1}^{2}$ is that of $u^{\prime \prime}(0)$, and these agree by the above formula for $u^{\prime \prime}(0)$. (Note incidentally that the height function for $u=0, v=0$ is right-equivalent at $t=0$ to $+t^{4}$ when $b_{4}>0$ and $-t^{4}$ when $b_{4}<0$.)

2. Pictures and examples for height functions. There are five cases in (1.7). The first three, where the projection is trivial, give uninteresting pictures for the dual: $k=1$ gives a smooth dual, $k=2$ a cusp edge and $k=3$ a swallowtail, independently of the value of $u$ close to 0 . (The critical values of the Gauss map are respectively empty, smooth and an ordinary cusp.)

The Morse case for $k=3$ gives the pictures of Fig. 1 for the evolution of the dual-compare [2, p. 31, Fig. 32(2)], or [1, p. 577, right].

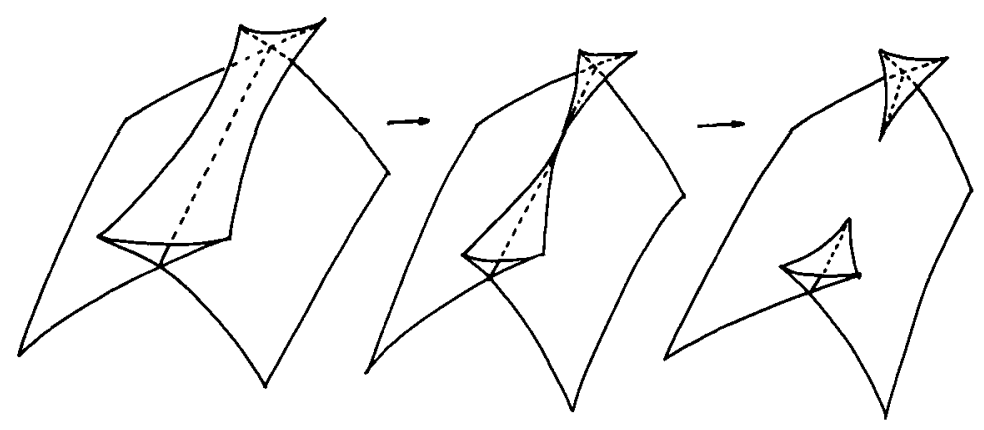

Figure 1.

The critical values of the Gauss map evolve by means of a "beaks" transition-Fig. 2. It is worth expanding a little on the geometry underlying these pictures. Recall that for $u=0$ (the central picture) the curvature of the space curve vanishes at $t=0$. When $\kappa(t) \neq 0$ there is a unique osculating plane at the point with parameter $t$, but when $\kappa(t)=0$ every tangent plane is osculating. Thus for $u \neq 0$ the dual, which models all the tangent planes,

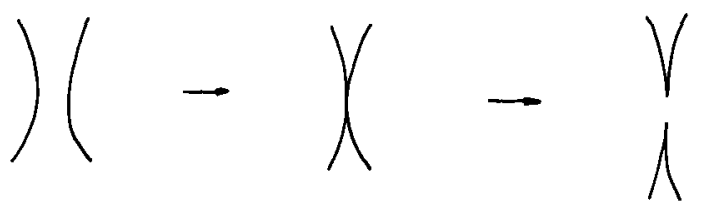

Figure 2. 
has a single circuit of cusp edge corresponding to the osculating planes at all points of the curve; it acquires an extra circuit of cusp edge for $u=0$, corresponding to the point of zero curvature. (We can suppose that, for $u$ close to 0 , no other point of zero curvature appears.)

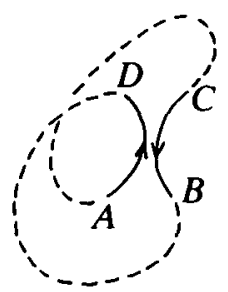

(a)

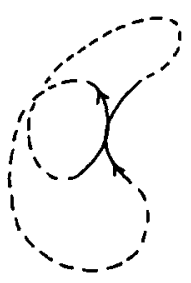

(b)

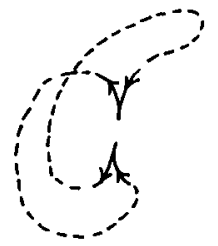

(c)

Figure 3.

In Fig. 3(a), $A$ must eventually be joined to $B$ or $C$ along the cusp edge, but in Fig. 3(c), if $A$ were joined to $B$ this would produce two circuits. Thus $A$ is joined to $C$ and the whole cusp edge has the form schematically drawn in Fig. 3, where the arrows come from an orientation of the space curve, and in 3(b) only one part can be oriented since the other circuit all comes from $t=0$.

The fundamental change here is that, in the sequence $(a) \rightarrow(b) \rightarrow(c)$, a pair of torsion zero points is born (corresponding to the cusps in (c)). But this happens via a $\kappa=0$ point (at which the torsion is undefined) rather than via a multiple zero of the torsion. One "half" of the circuit in 3(c) corresponds to the section of the space curve between the torsion zero points whose length tends to zero as $u \rightarrow 0$. When the length is very small we can treat the normal planes to the curve at points along this section as parallel, and can speak of the binormal direction as lying in a fixed plane. This direction then spins round very rapidly through an angle which approaches a multiple of $\pi$ as $u \rightarrow 0$. (In the example below it is $\pi$.)

As for bitangent planes (self-intersections of the dual) these form a curve in Fig. 1 which splits into two as $u$ passes through 0 . In the example which follows we work out how the points of contact are related to the torsion zero points.

We emphasise here (compare [4, p. 204]) that although we can produce all the local models for changes in the dual, Gauss map and focal set we do not have normal forms for the families $\Phi$ themselves. The examples which we give in (2.1), (2.2), (4.1) and (4.2) certainly exhibit the typical local changes as far as dual and Gauss map, or focal set, are concerned, and very likely they capture other typical aspects of the changing geometry as well. The general problem of saying what all the relevant geometry is, and producing normal forms typifying all the generic changes, appears to be difficult but interesting.

(2.1) Example. $\Phi(t, u)=\left(t, t^{3}, t^{4}+u t^{2}\right)$. This satisfies all the conditions given in $\$ 1$ for a generic isotopy in the $k=3$, Morse projection case. We have:

$$
F(t, v, u, w)=v_{1} t+v_{2} t^{3}+t^{4}+u t^{2}-w
$$


using the version of $F$ given following (1.3). The cusp edge on the dual is given by $F=\partial F / \partial t=\partial^{2} F / \partial t^{2}=0$; for a fixed $u$ this is the set of points

$$
\left(v_{1}, v_{2}, w\right)=\left(2 t^{3}-u t,-\left(6 t^{2}+u\right) / 3 t, t^{4}-u t^{2} / 3\right) \text {. }
$$

The torsion zero points on the curve give cusps on the cusp edge where $\partial^{3} F / \partial t^{3}=0$, namely where $v_{2}=-4 t$. This gives $t^{2}=u / 6$, so there are two such points when $u>0$ and none when $u<0$.

Note that the cusp edge goes to infinity: this is because our coordinates $\left(v_{1}, v_{2}\right)$ do not account for all of $S^{2}$. In fact this means that we have to be careful about deducing the binormal direction, since it is $\pm\left(v_{1}, v_{2}, 1\right) /\left(v_{1}^{2}+v_{2}^{2}+1\right)^{1 / 2}$ and it will be necessary to switch signs when $v$ goes to infinity if we are to follow a continuous movement of the binormal itself.

Thus, when $u>0$, as $t$ goes from $-(u / 6)^{1 / 2}$ to $+(u / 6)^{1 / 2}$ the $\left(v_{1}, v_{2}\right)$-curve obtained by projection of the cusp edge goes from $\left(\left(2 u^{3} / 27\right)^{1 / 2},(8 u / 3)^{1 / 2}\right)$ to $(0, \infty)$ and then from $(0,-\infty)$ to $\left(-\left(2 u^{3} / 27\right)^{1 / 2},-(8 u / 3)^{1 / 2}\right)$. As $u \rightarrow 0$ this approaches a closed curve with one excursion to infinity, but on the sphere it will approach a curve joining $(0,0,1)$ to $(0,0,-1)$ (or vice versa). This indicates that the binormal spins through an angle which approaches $\pi$, between the two torsion zero points on the curve.

Bitangent planes correspond to $\left(v_{1}, v_{2}, w\right)$ on the dual where $F=0, \partial F / \partial t=0$ has two solutions $t_{1}, t_{2}$. Thus $F$ has the form $\left(t-t_{1}\right)^{2}\left(t-t_{2}\right)^{2}$ which gives

$$
t_{1}^{2}+4 t_{1} t_{2}+t_{2}^{2}=u
$$

on comparing coefficients of $t^{2}$. This relationship between $t_{1}$ and $t_{2}$ is represented in Fig. 4. (Of course we are concerned with unordered pairs $\left\{t_{1}, t_{2}\right\}$, so Fig. 4 should be "factored" by reflexion in the line $t_{1}=t_{2}$.)

Note that $t_{1}=t_{2}$, for $u \geqslant 0$, gives the torsion zero points at $t_{1}= \pm(u / 6)^{1 / 2}$, and the points of contact of one bitangent plane have coincided. For each $t_{1}$ other than this value there are 0 or 2 values of $t_{2}$ giving a genuine bitangent plane. Following the fate of a particular $t_{1}$ and its corresponding $t_{2}$ 's giving bitangent planes we have something like Fig. 5 . For each $u$ there is on the self-intersection line of the dual a symmetrical $(2,2)$ correspondence. For $u<0$ this has four special points (at $t= \pm(-u / 3)^{1 / 2}, t= \pm 2(-u / 3)^{1 / 2}$ ) where there are coincidences among corresponding points. For $u=0$ only $t=0$ is special. For $u>0$ there are two special points (at $t= \pm(u / 6)^{1 / 2}$ ) which are self-corresponding and where the torsion is zero. These are visible on the dual as end-points of the self-intersection line. There are bitangent planes with their points of contact astride each torsion zero point on the curve.

We now turn to the $A_{4}$ transition of (1.7). The pictures of the dual are shown in Fig. 6 (compare [2, Fig. 32(5)] or [1, p. 577, left]). Because of its comparative complexity the last one is shown in an "exploded" form; the two cut off ends are to be identified according to the letters. The critical values of the Gauss map evolve through a swallowtail transition.

What is happening on the curve here is the coalescence and disappearance of two torsion zero points via a double zero of the torsion. This time there is always a unique 

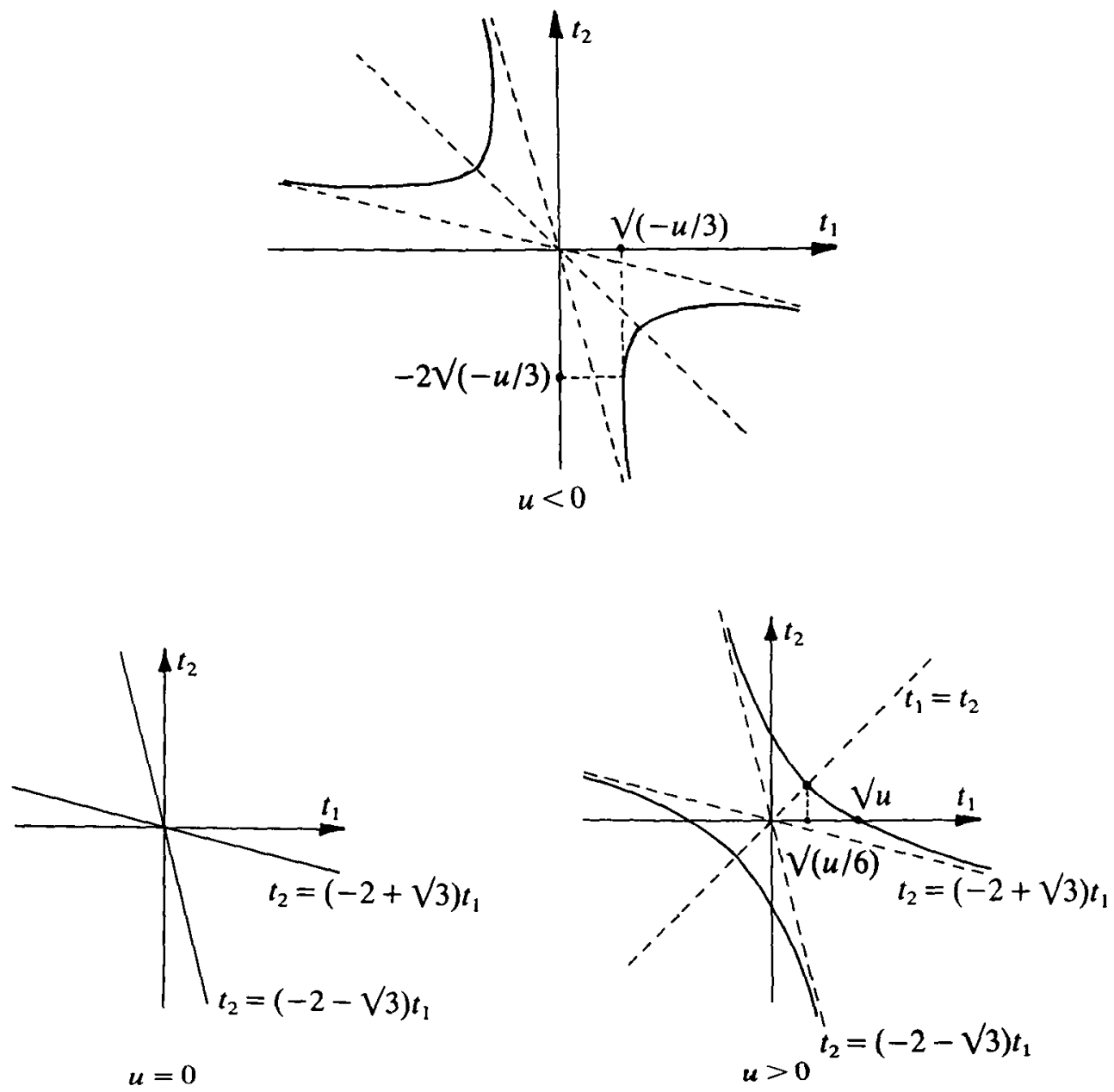

Figure 4 .

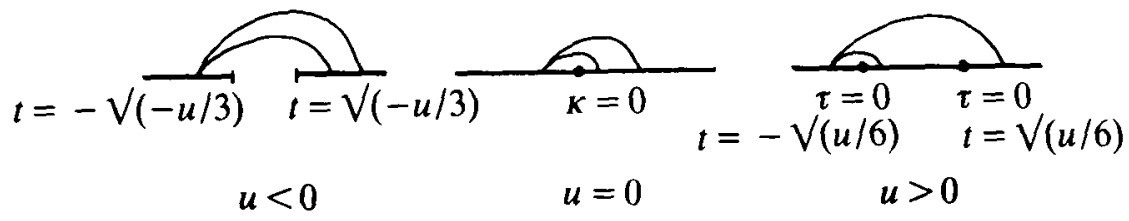

Figure 5 . 

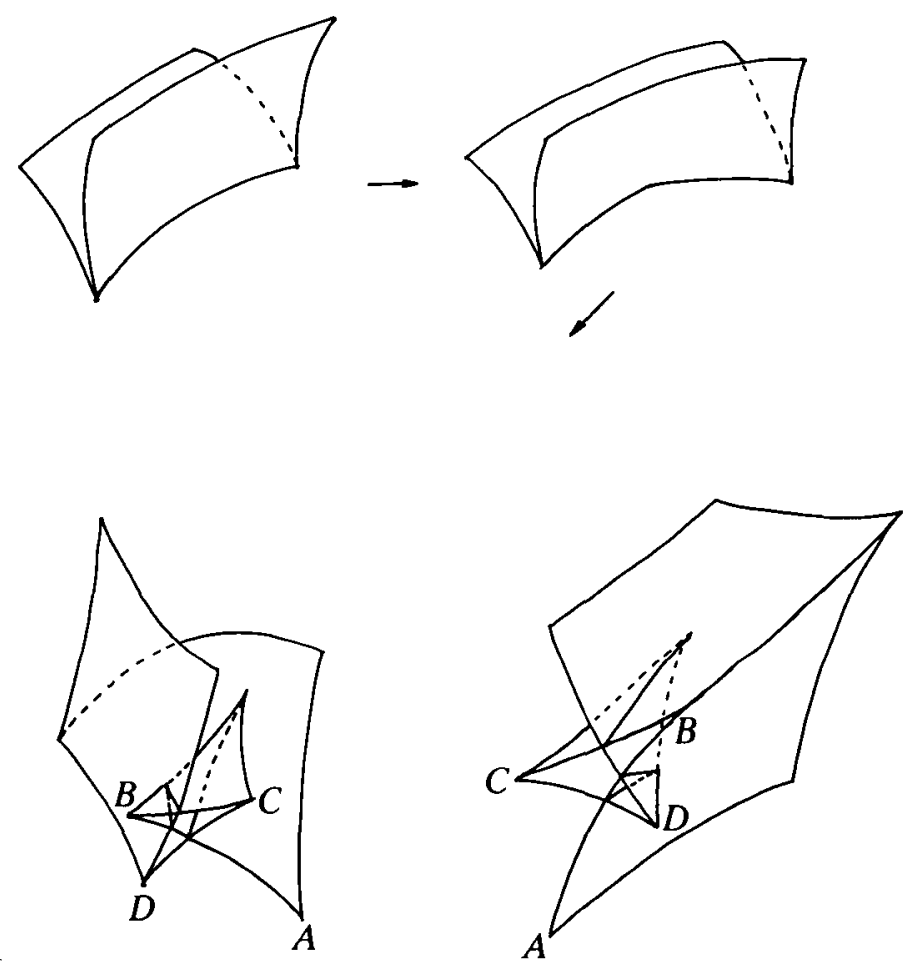

Figure 6.

osculating plane at each point of the curve and two cusps on the cusp edge of the dual come together and are annihilated.

(2.2)

$$
\begin{aligned}
\text { ExAmple. } & \Phi(t, u)=\left(t, t^{2}, t^{5}+u t^{3}\right) \\
& F\left(t, v_{1}, v_{2}, u, w\right)=v_{1} t+v_{2} t^{2}+t^{5}+u t^{3}-w
\end{aligned}
$$

The cusp edge of the dual, for fixed $u$, consists of points

$$
\left(15 t^{4}+3 u t^{2},-10 t^{3}-3 u t, 6 t^{5}+u t^{3}\right)
$$

and cusps on this have $u=-10 t^{2}$. Thus for $u<0$ there are two and for $u>0$ none. The calculation of bitangent planes is a little more complicated than before, but we find that $t_{1}$ and $t_{2}$ are the points of contact of such a plane if and only if

$$
3 t_{1}^{2}+4 t_{1} t_{2}+3 t_{2}^{2}=-u
$$

For $u>0$ this has no solutions and for $u=0$ only $t_{1}=t_{2}=0$. For $u<0$ the solutions form an ellipse as in Fig. 7. The points where $t_{1}=t_{2}$ correspond to ends of the self-intersection set on the dual, and are the torsion zero points on the curve. The self-intersection set itself has cusps (Fig. 6) which lie on the cusp edge of the dual; thus the corresponding 


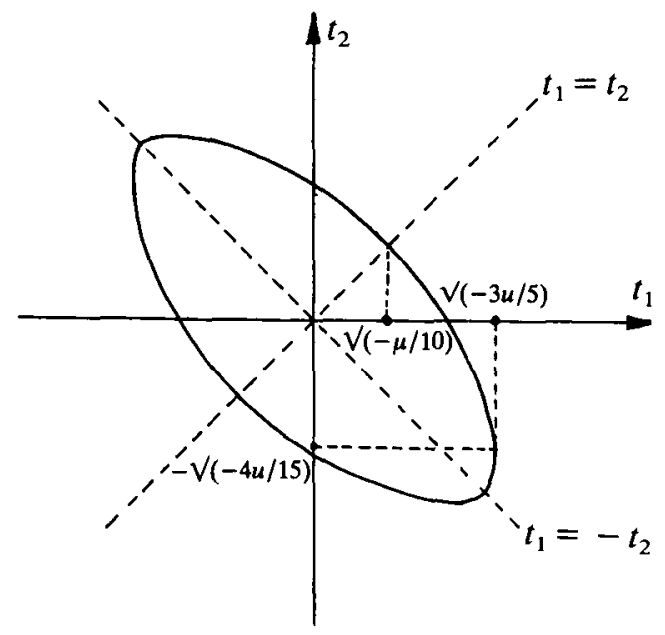

Figure 7.

bitangent plane is osculating at one of its points of contact. When $t_{1}$ has the extreme value $\pm(-3 u / 5)^{1 / 2}$ (Fig. 7) the values of $t_{2}$ coincide at $\mp 2(-37 / 5)^{1 / 2} / 3$ and the bitangent plane is osculating at $t_{2}$.

3. Distance-squared functions. We use the same notation for curves $\phi$ and isotopies $\Phi$ as in $\S 1$, but now consider the functions

$$
g: C \times \mathbb{R}^{3} \rightarrow \mathbb{R}
$$

and

$$
G: C \times U \times \mathbb{R}^{3} \rightarrow \mathbb{R}
$$

given by

$$
g(t, x)=\|\phi(t)-x\|^{2}
$$

and

$$
G(t, u, x)=\|\Phi(t, u)-x\|^{2}
$$

The bifurcation set of $g$,

$$
\mathscr{B}(g)=\left\{x: \partial g / \partial t=\partial^{2} g / \partial t^{2}=0 \text { at }(t, x) \text { for some } t\right\}
$$

is then the focal set of $\phi$ and the bifurcation set of $G$ is the "big focal set"; we are interested in the local structures of $\mathscr{B}(g)$ and $\mathscr{B}(G)$, and in the projection $\mathscr{B}(G) \rightarrow U$. One way of viewing this situation is to think of $\phi(C)$ as a light filament and the focal set as the resulting caustic. We are studying the way this caustic changes as the filament is moved about. 
For a single curve $\phi$, with curvature $K$ and torsion $\tau$, the condition for $g(-, x)$ to have type $A_{2}$ at $t$ is that $K(t) \neq 0$ and

$$
x=\phi(t)+N(t) / \kappa(t)+\mu B(t)
$$

$(N, B$ being principal normal and binormal), where

or

$$
\tau(t) \neq 0 \text { and } \mu \neq-\kappa^{\prime}(t) / K^{2}(t) \tau(t)
$$

$$
\tau(t)=0 \quad \text { and } \quad \kappa^{\prime}(t) \neq 0 .
$$

In either case we find that $g$ versally unfolds the singularity. The first alternative just says that $x$ is not the centre of spherical curvature at $t$. In the second case this centre has merely gone to infinity. For type $A_{3}$ or $A_{4}$ the conditions are complicated but (see [6, p. 124]) the singularities are versally unfolded, provided only $\tau(t) \neq 0$ for $A_{4}$ (a generic condition). We have the following result.

(3.1) Proposition. For a generic curve $\phi$ the focal set is, locally at $x$,

(i) smooth whenever $g(-, x)$ has type $A_{2}$ at $x$,

(ii) cusp edge whenever $g(-, x)$ has type $A_{3}$ at $x$,

(iii) swallowtail whenever $g(-, x)$ has type $A_{4}$ at $x$.

Now we turn to isotopies, using the notation of (1.2) and (1.3), and writing $\left(x_{1}, x_{2}, x_{3}\right)$ for $x$.

(3.2) $G(-, 0, x)$ has type $A_{\geqslant k}$ at $t$ if and only if

$$
\begin{aligned}
& k=1: x_{1}=0 ; \\
& k=2: \text { also } 1-2 x_{2} a_{2}-2 x_{3} b_{2}=0 ; \\
& k=3: \text { also } x_{2} a_{3}+x_{3} b_{3}=0 ; \\
& k=4: \text { also } a_{2}^{2}+b_{2}^{2}-2 x_{2} a_{4}-2 x_{3} b_{4}=0 ; \\
& k=5: \text { also } a_{2} a_{3}-x_{2} a_{5}+b_{2} b_{3}-x_{3} b_{5}=0 ; \\
& k=6: \text { also } a_{3}^{2}+2 a_{2} a_{4}-2 x_{2} a_{6}+b_{3}^{2}+2 b_{2} b_{4}-2 x_{3} b_{6}=0 .
\end{aligned}
$$

Write $\left(t-x_{1}\right) \psi_{1}(t)+\left(\phi_{1}(t)-x_{2}\right) \psi_{2}(t)+\left(\phi_{3}(t)-x_{3}\right) \psi_{3}(t)$ as a formal series $\rho_{0}+\rho_{1} t+$ $\rho_{2} t^{2}+\ldots$.

(3.3) The conditions for $G$ to be a versal unfolding of the function $G(-, 0, x)$ of type $A_{k}$, at $t=0$, are

$$
\begin{aligned}
& k \leqslant 3: \text { no condition; } \\
& k=4:\left\{\begin{array}{l}
\text { either }\left(a_{3} \neq 0 \text { or } b_{3} \neq 0\right) \\
\text { or } a_{3}=b_{3}=0, c_{2}+d_{1} a_{2}-d_{3} x_{2}+e_{1} b_{2}-e_{3} x_{3} \neq 0
\end{array}\right. \\
& k=5:\left|\begin{array}{lll}
a_{2} & a_{3} & a_{4} \\
b_{2} & b_{3} & b_{4} \\
\rho_{2} & \rho_{3} & \rho_{4}
\end{array}\right| \neq 0 .
\end{aligned}
$$


These are of course all straightforward but tedious verifications.

Finally we need the additional conditions for the projection $\mathscr{B}(S) \rightarrow U$ to be one of Arnold's generic functions. Since we are dealing with $A_{k}$ singularities the bifurcation set of an $A_{k}$ is diffeomorphic to the discriminant of an $A_{k-1}$, and the methods of $[1,3]$ apply. We need $k \geqslant 2$ for $\mathscr{B}(G)$ to be nonempty.

(3.4) $k=2$ or 3: projection always trivial.

$$
k=4:\left\{\begin{array}{l}
\text { trivial } \Leftrightarrow a_{3} \neq 0 \text { or } b_{3} \neq 0, \\
\text { Morse }\left(\sim \pm\left(\lambda_{1}+\tau_{1}^{2}\right)\right) \Leftrightarrow a_{3}=b_{3}=0, a_{2} b_{4} \neq a_{4} b_{2} .
\end{array}\right.
$$

(Equivalent to $\tau=\kappa^{\prime}=0, \tau^{\prime} \neq 0$ for the $u=0$ curve at $t=0$.)

$$
k=5: \text { standard } \Leftrightarrow a_{2} b_{3}-a_{3} b_{2} \neq 0 .
$$

The conditions $a_{3}=b_{3}=0$ (or $\tau=\kappa^{\prime}=0$ ) say that the line of points $x$ of the form $\phi(0)+N(0) / K(0)+\mu B(0)$ (where $\mu \in \mathbb{B}$, and $\phi(t)=\Phi(t, 0)$ is the $u=0$ curve) gives a singularity $A_{\geqslant 3}$ for $G(-, 0, x)$ at $t=0$. The condition $a_{2} b_{4} \neq a_{4} b_{2}$ says that there is nevertheless precisely one point of this line giving type $A_{\geqslant 4}$, namely the one with $\mu=\kappa^{\prime \prime} / \kappa^{2} \tau^{\prime}$ at $t=0$.

The proofs of the statements in (3.4) are similar to those in (1.7). Again, the fact that the Morse projection equivalent to $\pm\left(\lambda_{1}-\tau_{1}^{2}\right)$ does not occur follows geometrically: the cusp edge of the focal set contains the centres of spherical curvature, and these always exist. Thus, as for height functions, the cusp edge cannot vanish completely. An algebraic argument based on explicit reduction to normal form gives the same result.

4. Pictures and examples for distance-squared functions. The pictures are, naturally, the same as those in $\$ 2$, though since we are dealing with bifurcation sets an $A_{k}$ here has picture the same as $A_{k-1}$ in $\$ 2$. Thus the first three cases of (3.4) give focal sets which are smooth, cusp edge and swallowtail, independently of $u$.

Consider first the Morse case, $k=4$, for which the transition of focal sets is shown in Fig. 1. The cusp edge is the set of $A_{k}$ points for $k \geqslant 3$; this acquires an extra line when $u=0$, corresponding to the vanishing of both $\tau$ and $\kappa^{\prime}$ at $t=u=0$. For other values of $u$ close to 0 the cusp edge is connected, allowing for excursions to infinity at points where $\tau=0$ (but $\kappa^{\prime} \neq 0$ ). The direction in which the cusp edge goes to infinity for $t$ close to 0 provides, as $u \rightarrow 0$, the direction of the extra line which appears at $u=0$. The ways in which the cusp edge is connected up follow Fig. 3, but now the loops have points at infinity on them. The following example exhibits this transition; recall the remarks preceding Example 2.1 above.

(4.1) Example. $\Phi(t, u)=\left(t, t^{4}, t^{2}+t^{5}+u t\right)$. This satisfies all the conditions for a generic $A_{4}$ Morse transition. Thus $G(t, u, x)=\left(x_{1}-t\right)^{2}+\left(x_{2}-t^{4}\right)^{2}+\left(x_{3}-t^{2}-t^{5}-u t\right)^{2}$. The extra line which appears in the $A_{\geqslant 3}$ set of $G$ (cusp edge) for $u=0$ is the line $x_{1}=0$, $x_{3}=\frac{1}{2}$ and in this example $t=0$ always gives a point of zero torsion, where the cusp edge goes to infinity. Naturally the formulas for points $\left(x_{1}, x_{2}, x_{3}\right)$ on the cusp edge are rather complicated, but computer plots of the projection to $\left(x_{1}+x_{3}, x_{2}\right)$ look like Fig. 8 . 

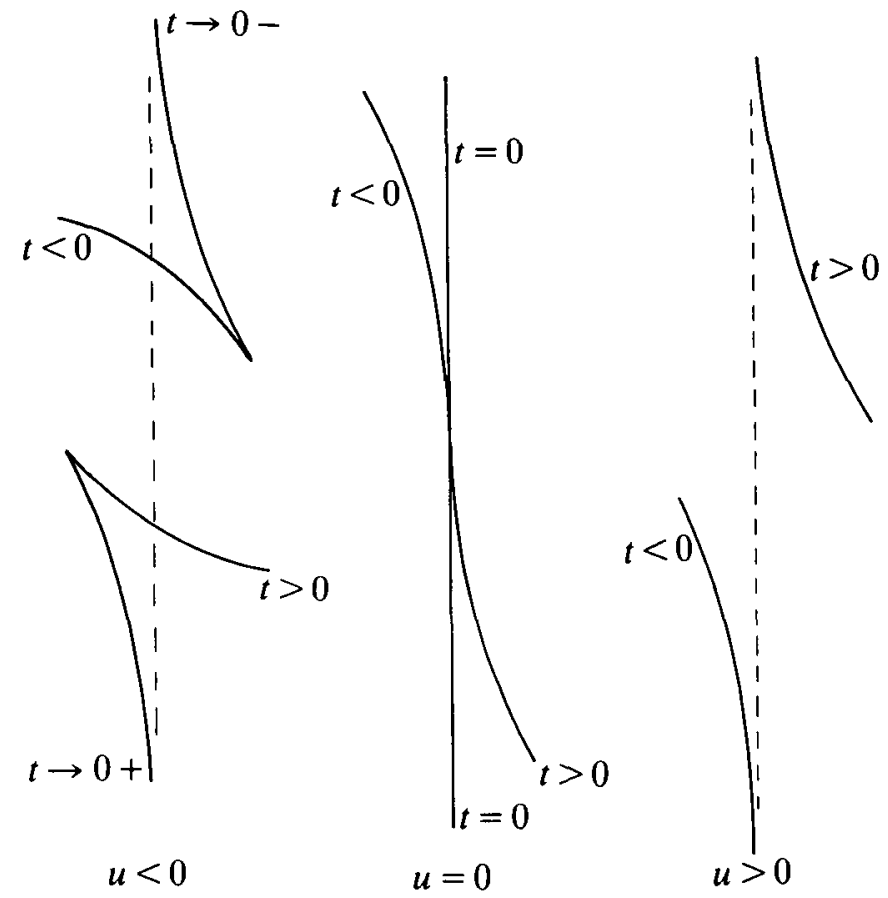

Figure 8 .

The self-intersection locus on the focal set does not have the geometrical interest that it does on the dual: it is the locus of points which are centres of two spheres touching the curve with 3-point $\left(A_{2}\right)$ contact at two different places, that is the $A_{2} / A_{2}$ locus. The end-points of this locus (Fig. 1, right) are centres of spheres having $A_{4}$ contact with the curve: the two different places of contact have coalesced.

The picture for the generic $A_{5}$ transition is Fig. 6 . Here two points of the curve giving swallowtail points $\left(A_{4}\right.$ points) coalesce - but, as was pointed out above, this does not have any direct connexion with the geometry of the curve apart from its contact with spheres. An example of a generic $A_{5}$ isotopy for distance-squared functions is

$$
\Phi(t, u)=\left(t, t^{2}+t^{4}, t^{3}+u t\right) .
$$

Here, the cusp edge $\left(A_{4}\right.$-set) of the focal surface has two cusps when $u<0$ and none when $u>0$. Thus in Fig. 6 the diagrams correspond respectively to $u>0, u=0, u<0$.

5. Multi-local phenomena. The dual of a space curve, being a surface in $\mathbb{R}^{3}$, will in general intersect itself. For instance if a single plane is osculating at one point of the curve and tangent at another, then we expect that a cusp edge on the dual will cross a smooth part of the dual at the corresponding point. Likewise a tritangent plane will give a triple crossing of smooth parts of the dual. How do these multi-local (or quasi-global) 
phenomena evolve as the curve moves through an isotopy? There will be isolated moments $u \in U$ when more degenerate situations, such as quadri-tangent planes, occur, and it is geometrically rather clear what we can expect to occur on the evolving duals.

In this section we show how to handle this situation and to find explicitly the conditions for multi-local transitions to have the expected generic forms. Calculation shows that nothing unexpected happens, so we do not go into great detail, giving the actual genericity calculation only in one case ((5.4) below). Our methods are based on [5] and apply to any situation where there are intersections of discriminants of $A_{k}$ singularities for various $k$, but the example to keep in mind is that of the big family $F$ in $\$ 1$ associated to height functions. In that case the degenerate phenomena which occur generically at isolated moments of the isotopy turn out to be the following.

\begin{tabular}{ll}
\hline Singularities of $F$ & \multicolumn{1}{c}{ Geometrical interpretation } \\
\hline$A_{1}^{4}=A_{1} A_{1} A_{1} A_{1}$ & Plane tangent at 4 points. \\
$A_{1}^{2} A_{2}$ & Plane tangent at 2 points, osculating at a third. \\
$A_{2} A_{2}$ & Plane osculating at two points. \\
$A_{1} A_{3}$ & $\begin{array}{l}\text { Plane tangent at one point, osculating at } \\
\text { another, where } \tau=0 .\end{array}$ \\
$A_{1}^{3}$ & Plane tangent at three collinear points. \\
$A_{1} A_{2}$ & Plane tangent at one point and osculating \\
& at a second, the first lying on the tangent \\
$A_{1}^{2}$ & line at the second. \\
\hline
\end{tabular}

The last three cases are all "Morse transitions" where the projection to the $U$ parameter of the intersecting discriminants has a Morse component-in the normal form of [5] a square $\tau_{1}^{2}$ for $A_{1}^{3}$ and $A_{1} A_{2}$ and a sum $\tau_{1}^{2} \pm \tau_{2}^{2}$ for $A_{1}^{2}$. In fact the + and - are distinguishable by the presence or absence of bitangent plane: the + sign occurs precisely when the two branches of the curve are locally on opposite sides of the plane spanned by the tangent lines at the node. Of course $A_{1}^{2}$ is very special in another way: it could not possibly occur during an isotopy of embedded curves! Presumably it is possible to relax the definition of isotopy so that parts of the curve close to two parameter values can be moved independently, but we do not go into such modifications here.

We start with the case of a point stratum, that is for $F$ one of the first 4 cases in (5.1). We recall some results from [5] for functions on unions of transversally intersecting $A_{k}$ discriminants.

Let $P_{i}: \mathbb{R} \times \mathbb{R}^{k(i)}, 0 \rightarrow \mathbb{R}, 0$ be, for $1 \leq i \leq r$, the germ

$$
P_{i}\left(t, a^{i}\right)=t^{k(i)+1}+a_{1}^{i} t^{k(i)-1}+\ldots+a_{k(i)}^{i},
$$

where we write $a^{i} \in \mathbb{R}^{k(i)}$ as $\left(a_{1}^{i}, \ldots, a_{k(i)}^{i}\right)$. Thus $P_{i}$ is a miniversal unfolding of a germ of type $A_{k(i)}$. The germs $P_{i}$ give a multigerm $P: \mathbb{R} \times \mathbb{R}^{k} \rightarrow \mathbb{R}\left(k=\sum k_{i}\right)$, defined on neighbourhoods of points $t_{1}, \ldots, t_{r}$ in $\mathbb{R}$ by $P\left(t_{i}+t, a^{1}, \ldots, a^{r}\right)=P_{i}\left(t, a^{i}\right)$ for $t$ close to 0 . 
The discriminant $\mathscr{D}(P)$ is then

$$
\mathscr{D}(P)=\left\{\left(a^{1}, \ldots, a^{r}\right) \in \mathbb{R}^{k}: \text { for some } i \text { and } t, P_{i}=\partial P_{i} / \partial t=0 \text { at }\left(t, a^{i}\right)\right\} .
$$

This is the union of $r$ "discriminant cylinders" in $\mathbb{P}^{k}$, each of the form

$$
\text { (discriminant of } \left.A_{k(i)}\right) \times \mathbb{R}^{k-k(i)} \text {. }
$$

In [5] one of us classified functions on such standard discriminants. In fact if $p: \mathbb{R}^{k}, 0 \rightarrow \mathbb{R}, 0$ satisfies $\partial p / \partial a_{1}^{i}(0) \neq 0$ for $i=1, \ldots, r$ then $p$ is stable as a function on $\mathscr{D}(P)$ and equivalent to $\sum_{i=1}^{r} \varepsilon_{i} a_{1}^{i}$ where each $\varepsilon_{i}$ is \pm 1 . In order to apply this to a situation where the unfoldings and discriminant are not in such a convenient normal form, we need to interpret the conditions in an invariant way.

Suppose then that we have a family of functions

$$
F: M \times A \times U \rightarrow \mathbb{R}
$$

where $M$ is a manifold (for us a closed curve in $\mathbb{R}^{3}$ ), $A$ is a parameter space and $U$ is the one-dimensional "time parameter" space. We are thinking particularly of the $F$ of $\$ 1$, where $A=S^{2} \times \mathbb{R}$, but the method is general, and of course easily adapted to cover bifurcation sets of multigerms having $A_{k}$ singularities. Write $F_{a, u}$ for the function $F(-, a, u)$. The discriminant $\mathscr{D}(F)$ is

$$
\mathscr{D}(F)=\left\{(a, u) \text { : for some } t \in M, T F_{a, u}(t): M_{t} \rightarrow \mathbb{R} \text { is zero and } F(t, a, u)=0\right\} .
$$

Now fix $a=a_{0}, u=u_{0}$ and suppose that $F_{a_{0}, u_{0}}$ has a singularity of type $A_{k(i)}$ at $t_{i} \in M$, for $i=1, \ldots, r$ (and no other singularity), with $F\left(t_{i}, a_{0}, u_{0}\right)=0$ for each $i$.

We need to write down the condition that the discriminant $\mathscr{D}(F)$ is near $\left(a_{0}, u_{0}\right)$, a transversally intersecting collection of discriminants of versal unfoldings of $A_{k(i)}$ singularities-and so, when $\sum k(i)=\operatorname{dim}(A \times U)$, diffeomorphic at $\left(a_{0}, u_{0}\right)$ to the standard model $\mathscr{D}(P)$ above. This condition is the natural multitransversality condition, as follows. Let $L$ be the submanifold of the multijet space ${ }^{K} J^{K}(M, \mathbb{R})(K>\max k(i))$ consisting of $r$-tuples $\left(j_{1}, \ldots, j_{r}\right)$ with each $j_{i}$ of type $A_{k(i)}$ and all constant terms zero. Then we require the following.

(5.2) The multijet extension

$$
j F={ }_{r} J^{K} F: M^{(r)} \times A \times U \rightarrow{ }_{r} J^{K}(M, \mathbb{R})
$$

is transverse to $L$ at $\left(t_{1}, \ldots, t_{r}, a_{0}, u_{0}\right)$. F.

Note that this automatically implies that all the singularities are versally unfolded by

We now assume $\sum k(i)=\operatorname{dim}(A \times U)$ and $(5.2)$, so that $(j F)^{-1}(L)$ is the single point $\left(t_{1}, \ldots, t_{r}, a_{0}, u_{0}\right)$. Write $N_{i}$ for the submanifold of ${ }_{r} J^{K}(M, \mathbb{R})$ for which all jets have constant term zero, $j_{l}$ has type $A_{k(l)}$ for $l \neq i$, and $j_{i}$ is in the closure of the $A_{k(i)-2}$ orbit. (This closure appears as $N\left(A_{k(i)}\right)$ in [3, p. 324]. For $k(i)=2$ this just requires zero constant term; for $k(i)=1$ it is no condition.) 
(5.3) Proposition. The projection

$$
\pi: \mathscr{D}(F),\left(a_{0}, u_{0}\right) \rightarrow U, u_{0}
$$

is equivalent to one of the stable functions on $\mathscr{D}(P)$ listed above provided the restricted multijet map

$$
j_{0} F: M^{(r)} \times A \times\left\{u_{0}\right\},\left(t_{1}, \ldots, t_{r}, a_{0}, u_{0}\right) \rightarrow J^{K}(M, \mathbb{R})
$$

is, for each $i$ (and all sufficiently large $K$ ), transverse to $N_{i}$.

Proof. The idea is, for each $i$, to find a smooth manifold in $A \times U$ at $\left(a_{0}, u_{0}\right)$ which meets $\mathscr{D}(F)$ in the discriminant of a miniversal unfolding of an $A_{k(i)}$ singularity. The condition for stability is then (using [3]) that the projection to $U$ of each of these discriminants is stable (this corresponds to each $\partial p / \partial a_{1}^{i}(0) \neq 0$ in the standard situation), and these conditions, using [3, Proposition 1.2], follow from those of the above proposition. Write $(j F)_{i}$ for the $i$ th component of $j F$ (regarding $J^{K}(M, \mathbb{R})$ as, locally, the product of $r$ copies of $\left.J^{K}(M, \mathbb{R})\right)$ and let $L_{i} \in J^{K}(M, \mathbb{R})$ consist of jets of type $A_{k(i)}$ with zero constant term. Thus, by (5.2), $j F$ is transverse to $L_{1} \times \ldots \times L_{r}=L$. Finally write $Q_{i}$ for $L_{i} \times \ldots \times L_{i-1} \times J^{K} \times L_{i+1} \times \ldots \times L_{1}$. Then it is not hard to see that

(1) $j F$ is transverse to $Q_{i}$,

(2) $(j F)_{i} \mid(j F)^{-1}\left(Q_{i}\right)$ is transverse to $L_{i}$,

(3) $(j F)_{i}^{-1}\left(L_{i}\right) \cap(j F)^{-1}\left(Q_{i}\right)=(j F)^{-1}(L)$.

It is (2) which assures us that each $A_{k(i)}$ is versally unfolded by $F$.

Now projecting $(j F)^{-1}\left(Q_{i}\right)$ to the parameter space $A \times U$ gives a smooth manifold $S_{i}$ say, of dimension $k(i)$, which meets the part of $\mathscr{D}(F)$ corresponding to the $i$ th copy of $M$ (points giving a singularity with zero value for $F$ near $t_{i}$ ) in the discriminant $\mathscr{D}_{i}$ of a miniversal unfolding of an $A_{k(i)}$ singularity. In Fig. $9 A \times U$ is of dimension 3 so that we can draw something. It is the projections $\left(S_{i}, \mathscr{D}_{i}\right) \rightarrow \mathbb{R}$ given by $(a, u) \rightarrow u$, which have to be stable maps on discriminants, the local splitting $A \times U=S_{1} \times \ldots \times S_{r}$ corresponding with the splitting $\mathbb{R}^{k(1)} \times \ldots \times \mathbb{R}^{k(r)}$ of the standard example $P$ above.
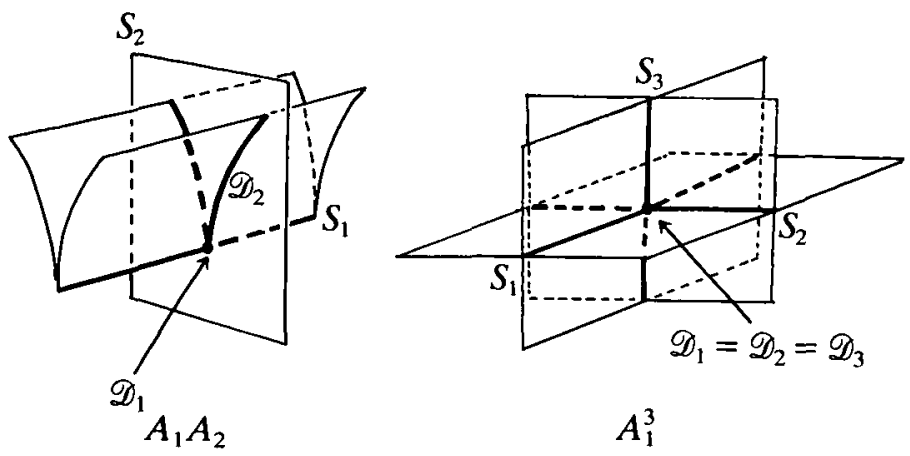

Figure 9. 
It remains to identify this condition with that in the proposition. In fact let $S_{i 0}$ be $\left\{\left(a, u_{0}\right)\right\} \in S_{i}$ and consider the composite

$$
M \times S_{i 0} \stackrel{\alpha}{\rightarrow} M^{(T)} \times A \times\left\{u_{0}\right\} \stackrel{j_{0} F}{\rightarrow}{ }_{r} J^{K}(M, \mathbb{R}) \stackrel{\pi_{i}}{\rightarrow} J^{K}(M, \mathbb{R}) .
$$

Here $\alpha\left(t, a, u_{0}\right)=\left(t_{1}, \ldots, t_{i-1}, t, t_{i+1}, t_{r}, a, u_{0}\right)$ and $\pi_{i}$ is projection to the $i$ th factor. The condition of the proposition is $j_{0} F$ 开 $N_{i}$ (for all $i$ ). Note that $M \times S_{i}$ can be identified locally with $(j F)^{-1}\left(Q_{i}\right)$-in fact the projection $(j F)^{-1}\left(Q_{i}\right) \rightarrow M \times A \times U$ (ignoring $M$ coordinates near $t_{j}$ for $\left.j \neq i\right)$, is an immersion at $\left(t_{1}, \ldots, t_{r}, a_{0}, u_{0}\right)$ and its image is $M \times S_{i}$. Likewise $M \times S_{i 0}$ can be identified locally with $\left(j_{0} F\right)^{-1}\left(Q_{i}\right)$, i.e., $\alpha\left(M \times S_{i 0}\right)=$ $\left(j_{0} F\right)^{-1}\left(Q_{i}\right)$. Using this it is an easy exercise in linear algebra to check that the above composite is transverse to $\pi_{i}\left(N_{i}\right)$, which is the $N\left(A_{k(i)}\right)$ of [3, Proposition 1.2]. Thus the criterion for a stable function on $\mathscr{D}_{i}$ is satisfied.

When we consider cases where $\operatorname{dim}(A \times U)-\sum k(i)=n>0$ then there are, as in [3], two kinds of conditions to check. The stratum $(j F)^{-1}(L)$ is then locally $\mathbb{R}^{n}$ and we have to check (i) that $\pi$ restricted to a transversal to this $\mathbb{R}^{n}$ in $A \times U$ satisfies the condition of (5.3), and (ii) that $\pi$ restricted to this $\mathbb{R}^{n}$ has a Morse singularity. Of course there are problems caused by the fact that the $\mathbb{R}^{n}$ and its transversal will present themselves as submanifolds of $A \times U$ in a less than transparent way. Rather than give all the details of a general method we present an example.

(5.4) Example. With the $F$ of $\S 1$ we consider the case of $A_{1} A_{2}$, so that $n=1$ above. The standard discriminant in $\mathbb{R}^{4}$ with coordinates $(a, b, c, d)$ can be written as $\left\{(a, b, c, d): a=0\right.$ or $\left.b^{3}+c^{2}=0\right\}$ and the stable Morse projection is $(a, b, c, d) \rightarrow a+$ $\varepsilon_{1} b+\varepsilon_{2} d^{2}$ where $\varepsilon_{1}, \varepsilon_{2}$ are \pm 1 . Examining the fibres of this projection and their intersections with the discriminant gives one of the two pictures of Fig. 10, according as $\varepsilon_{1} \varepsilon_{2}$ is +1 or -1 . Note that in Fig. 10(a) the central diagram $(u=0)$ shows that there are (locally) no bitangent planes other than the one giving rise to the $A_{1} A_{2}$ singularity pair, whereas in Fig. 10(b) the central diagram shows that there are other bitangent planes arbitrarily close by. The fact that the tangent plane to the smooth part of the dual contains the tangent line to the cusp edge is interpreted on the original curve in the statement of (5.1)-this is because the tangent line to the cusp edge is dual to the tangent line to the original curve.

For a multi-local calculation we can take the $u=0$ curve to have two branches

$$
\left(s, b_{2} s^{2}+\ldots, c_{3} s^{3}+\ldots\right) \text { and }\left(1+t, d_{1} t+\ldots, e_{2} t^{2}+\ldots\right)
$$

for small $s$ and $t$, where $c_{3} \neq 0$ and $e_{2} \neq 0$ so the singularities are $A_{2}, A_{1}$ and for simplicity we have taken the second branch to pass through $(1,0,0)$. Furthermore, we can change parameters so that the isotopies of the two branches are $\Phi(s, u)=\left(s, \Phi_{2}(s, u), \Phi_{3}(s, u)\right)$ and $\Theta(t, u)=\left(t, \Theta_{2}(t, u), \Theta_{3}(t, u)\right)$ for small $s, t, u$. We take height functions in directions $\left(v_{1}, v_{2}, 1\right)$.

For the multitransversality condition (5.2) it is enough to consider

$$
j: \mathbb{R}^{2} \times \mathbb{R}^{2} \times \mathbb{R} \times \mathbb{R} \rightarrow J^{2}(1,1) \times J^{1}(1,1)
$$




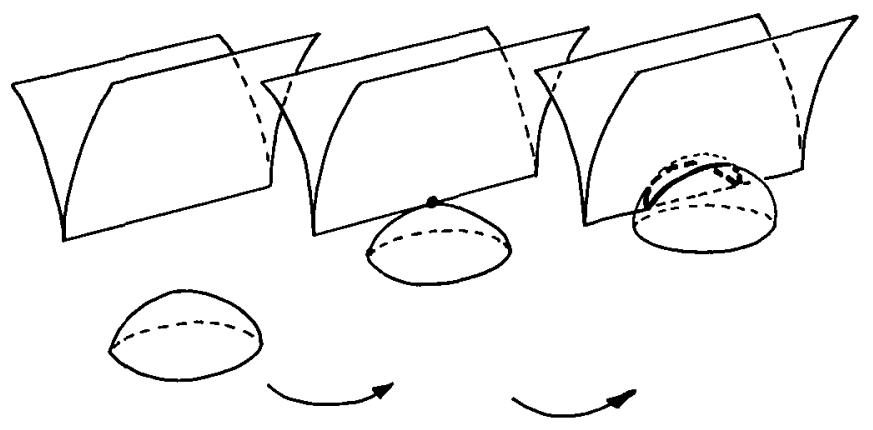

Figure 10(a).

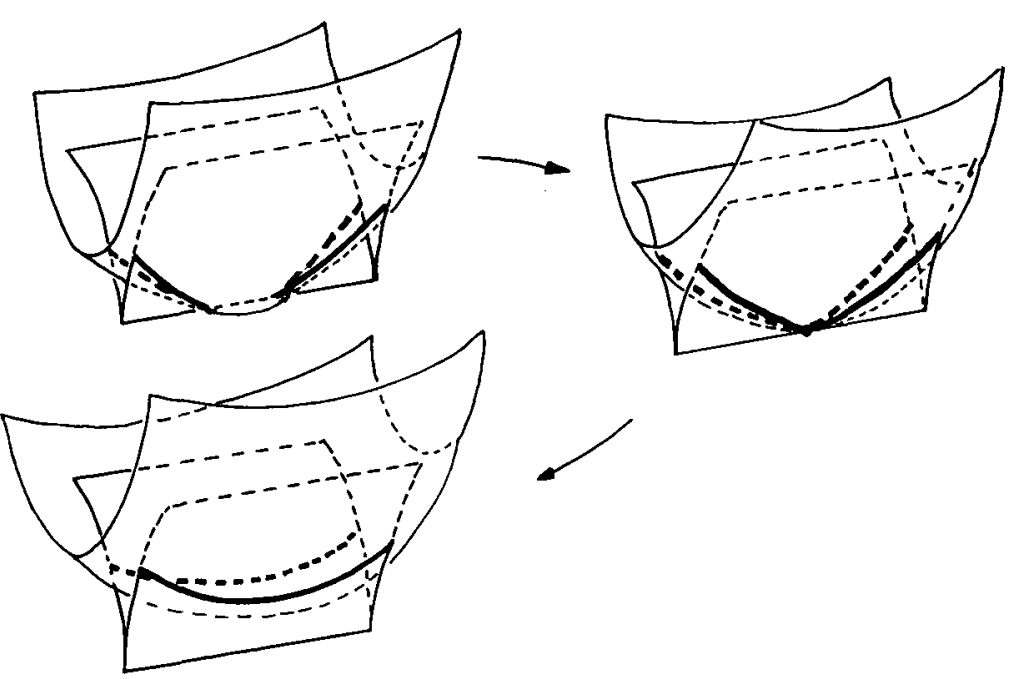

Figure 10(b).

given by $\left(s, t, v_{1}, v_{2}, u, w\right) \rightarrow\left(j_{1}, j_{2}\right)$ where $j_{2}$ is the 2-jet with constant at $s$ of $\Phi(s, u) \cdot\left(v_{1}, v_{2}, 1\right)-w$ and $j_{1}$ is the 1-jet with constant at $t$ of $\Theta(t, u) \cdot\left(v_{1}, v_{2}, 1\right)-w$. We require transversality at 0 to the $A_{2} A_{1}$-set in $J^{2} \times J^{1}$ which is $\{(0,0)\}$. In fact writing down the Jacobian matrix of $j$ we find that $v_{2}$ is a local coordinate on $j^{-1}(0,0)$ and on the projection $L$ of this to the parameter space $\mathbb{B}^{4}$ (coordinates $\left(v_{1}, v_{2}, u, w\right)$ ) provided

$$
\frac{\partial \Phi_{3}}{\partial u}(0) \neq \frac{\partial \Theta_{3}}{\partial u}(0)-\frac{\partial^{2} \Phi_{3}}{\partial s} \partial u(0)
$$

If (1) fails then $j$ is not a submersion at 0 , so (1) is the multitransversality condition, and a transversal to the $A_{2} A_{1}$ set $L$ in $\mathbb{R}^{4}$ is the $v_{2}=0$ plane, provided (1) holds.

Next we restrict $j$ to the plane $v_{2}=0$ and check the condition of (5.3), that is we use 
the restriction

$$
j_{0}: \mathbb{R}^{2} \times \mathbb{R} \times \mathbb{B} \rightarrow J^{2} \times J^{1}
$$

with coordinates $s, t, v_{1}, w$ on the left. Write $\alpha_{0}, \alpha_{1}, \alpha_{2}$ for the coefficients in the 2 -jet and $\beta_{0}, \beta_{1}$ for those in the 1 -jet. Then we need to check transversality of $j_{0}$ at 0 to

$$
N_{1}=\left\{\left(j_{1}, j_{2}\right): \alpha_{0}=\beta_{0}=\beta_{1}=0\right\}
$$

and

$$
N_{2}=\left\{\left(j_{1}, j_{2}\right): \alpha_{0}=\alpha_{1}=\alpha_{2}=0\right\} .
$$

These are easily checked to be automatic.

Finally we need to consider the map $L \rightarrow U$ given by projection to the $u$-coordinate and find the condition for this to be a Morse function. In fact we can equally well use $j^{-1}(0) \rightarrow U$, since $j^{-1}(0) \rightarrow L$ is an immersion. Using the local coordinate $v_{2}$ on $j^{-1}(0)$, so that $s, t, v_{1}, u$ and $w$ are all functions of $v_{2}$, we find $s^{\prime}(0)=-b_{2} / 3 c_{3}, t^{\prime}(0)=-d_{1} / 2 e_{2}$, $v_{1}^{\prime}(0)=0, u^{\prime}(0)=0, w^{\prime}(0)=0$. Further calculation shows that

$$
u^{\prime \prime}(0)\left(\frac{\partial \Theta_{3}}{\partial u}(0)-\frac{\partial \Phi_{3}}{\partial u}(0)-\frac{\partial^{2} \Phi_{3}}{\partial s \partial u}(0)\right)=\frac{d_{1}^{2}}{2 e_{2}}-\frac{2 b_{2}^{2}}{3 c_{3}}
$$

and since the expression in brackets is non-zero by (1) the other condition for a stable function is $u^{\prime \prime}(0) \neq 0$, i.e.

$$
3 d_{1}^{2} c_{3}-4 b_{2}^{2} e_{2} \neq 0
$$

Unfortunately (2) does not appear to have a simple geometrical interpretation. However it is easy to distinguish the two cases of Fig. 10 by calculating the condition for the existence of bitangent planes (i.e. coplanar tangent lines) to the two branches close to $s=t=0$. In fact we have Fig. 10(a) or (b) according as the left hand side of (2) is $>0$ or $<0$.

6. A transversality theorem. We shall use the same method as that of $[4, \S 3]$ to show that, generically, isotopies of space curves give rise only to the transitions of the dual, critical values of the Gauss map and focal set detailed in $\$ \$ 1-4$ above. In order to apply the "Monge-Taylor technique" of [4] (compare also [6, Chapter 9]) we need to set up a local coordinate system at each point of each curve in the isotopy. Unfortunately the standard triad of tangent, principal normal, binormal will not do, since we must allow for curves which have points of zero curvature, at which the principal normal is not defined. (Indeed, the example of (2.1) above illustrates rather graphically why it is hopeless to extend principal normals to points of zero curvature-recall how the binormal spins between the two $\tau=0$ points.)

Let $\phi: C \rightarrow \mathbb{R}^{3}$ be an oriented space curve. To each $t \in C$ assign a unit normal vector $N(t)$ to $\phi(C)$ at $\phi(t)$, making $t \mapsto N(t)$ smooth. We want to extend this "normal field" from one curve in an isotopy to all of them. Let $\omega: \mathbb{R}^{3} \rightarrow \mathbb{R}^{3}$ be a diffeomorphism; then $\omega \circ \phi$ is an oriented space curve and certainly $D \omega(\phi(t))(N(t))$ will be non-zero and not 
tangent to the curve $\omega \circ \phi$ at $\omega \circ \phi(t)$. Thus projecting this non-zero vector to the normal plane of $\omega \circ \phi$ at $\omega \circ \phi(t)$ and dividing by its length, we get a uniquely defined unit normal vector $N_{\omega}(t)$, which equals $N(t)$ when $\omega$ equals the identity. Completing "tangent, normal" to a right handed triad $T_{\omega}(t), N_{\omega}(t), \bar{N}_{\omega}(t)$ provides a uniquely defined triad at each point of each curve of an isotopy given by $\Omega: \mathbb{R}^{3} \times U \rightarrow \mathbb{R}^{3}$, once we assign $N$ to the curve $u=0$. The triad varies smoothly with $u$ and $t$.

Write $V_{p}^{k}$ for the real vector space of polynomials in $t$ of degree $\geqslant p$ and $\leqslant k$. Then for any family $\Phi$ as in (1.2) which is induced by an isotopy $\Omega$ there is a smooth map

$$
\Gamma: C \times U \rightarrow V_{2}^{k} \times V_{2}^{k} \times V_{0}^{k} \times V_{0}^{k} \times V_{0}^{k}
$$

defined as follows. Given $\left(t_{0}, u_{0}\right) \in C \times U$ choose axes at $\Phi\left(t_{0}, u_{0}\right)$ along the $T, N, \bar{N}$ directions as above, and by a change of the $t$ variable write $\Phi\left(t, u_{0}\right)=\left(t, \phi_{2}(t), \phi_{3}(t)\right)$ for $t$ close to $t_{0}$. The first two components of $\Gamma$ are the $k$-jets of $\phi_{2}, \phi_{3}$ at $t_{0}$. For the other three components we use the $k$-jets at $t_{0}$ of

$$
\left.\frac{\partial \Phi_{i}}{\partial s}\left(t, u_{0}+s\right)\right|_{s=0}
$$

for $i=1,2,3$. (Compare (1.3).)

Of course $\Gamma$ depends not only on the isotopy but on the choice of normal field $N$. However, we shall only be considering geometrically defined subsets of the target of $\Gamma$, which will be invariant under a change of $N$. By using as deformations maps $\mathbb{R}^{3} \rightarrow \mathbb{R}^{3}$ whose components are polynomials of degree $\leqslant k$, one proves the following in exactly the same way as for Theorem 3.2 of [4].

(6.1) THEOREM. Let $X$ be a Whitney (A) regular stratified subset of $\left(V_{2}^{k}\right)^{2} \times\left(V_{0}^{k}\right)^{3}$, and let $\Phi$ be an isotopy between the generic curves (see note below) $C_{0}$ and $C_{1}$. Then there is an arbitrarily small deformation of $\Phi$ to an isotopy between the same curves $C_{0}$ and $C_{1}$ for which the corresponding map $\Gamma$ is transverse to $X$.

NoTEs. (1) In the applications, "generic curve" can be given a specific meaning: in fact when we are concerned with height functions it has the usual meaning that the family of height functions $h$ on the curve should have jet extension transverse to the natural stratification of $J^{k}(1,1)$, for all $k$ sufficiently large. Thus, as in (1.1), only singularities $A_{\leqslant 3}$ appear and they are versally unfolded by $h$. Similarly, with distance-squared functions (compare (3.1)).

(2) The only essential difference between the situation here and that in [4] is that, here, we need our isotopies to be given by families of diffeomorphisms of $\mathbb{R}^{3}$ in order to carry over the choice of normal vectors from one curve to another. Fortunately all the constructions of the proof of $[4, \S 3.2]$ use explicitly diffeomorphisms of balls in $\mathbb{R}^{3}$, which can of course be extended to diffeomorphisms of the whole of $\mathbb{R}^{3}$. Thus at every stage the normal vectors to all the curves are well-defined.

When we apply (6.1) we need to specify the set $X$, which represents some conditions on the isotopy we want to avoid, and to check that $X$ is semi-algebraic of codimension at least 3. (The A-regularity condition is then automatic.) The transversality of $\Gamma$ to $X$ then 
ensures that the image of $\Gamma$ misses $X$ : the nearby isotopy does avoid all the unwanted conditions. This will then prove that such isotopies are dense, and openness will always be automatic. In this way we prove that isotopies avoiding the chosen conditions are generic, that is open and dense.

First consider the height functions. Given $\left(t_{0}, u_{0}\right) \in C \times U$ we choose axes as specified above; the only problem is that generally the direction for the height function will not be along the third $(\bar{N})$ axis. However for a singular height function (the only case of interest) the direction will be in the normal plane at $\Phi\left(t_{0}, u_{0}\right)$, so by a rotation of axes in this plane we can use the formulas of (1.4), (1.5) and (1.7). Before rotation the subset of $\left(V_{2}^{5}\right)^{2} \times\left(V_{0}^{5}\right)^{3}$ corresponding to bad isotopies is given by

$$
\begin{gathered}
a_{2}=a_{3}=b_{2}=b_{3}=0 \text { or } a_{2}=b_{2}=b_{3}=e_{2}=0 \\
\text { or } a_{2}=b_{2}=b_{3}=b_{4}=0 \text { or } b_{2}=b_{3}=b_{4}=a_{2} e_{3}-a_{3} e_{2}=0 \\
\text { or } b_{2}=b_{3}=b_{4}=b_{5}=0,
\end{gathered}
$$

using the $a, \ldots, e$ coordinates as in (1.3). This is algebraic of codimension 4 , and the effect of introducing rotations is to make it semi-algebraic of codimension at least 3 . Thus the set $X$ to be avoided for height functions has $k=5$ and is semi-algebraic of codimension $\geqslant 3$. Thus we have the following result.

(6.2) THEOREM. The isotopies, for which all transitions on the dual and the critical values of the Gauss map are of the four types in (1.7), are generic (open and dense).

For distance-squared functions the situation is very similar, except that here the bad set is initially a subset of $\mathbb{B}^{3} \times\left(V_{2}^{6}\right)^{2} \times\left(V_{0}^{6}\right)^{3}=\mathbb{R}^{5} \times V$, say, since the conditions of (3.2) and (3.3) involve the $\left(x_{1}, x_{2}, x_{3}\right)$-coordinates in $\mathbb{R}^{3}$. However each case that we want to avoid certainly gives an algebraic subset $Y$ of this bigger space whose projection $X$ to $V$ is a semi-algebraic set. Since projection cannot increase dimension we need only check that $Y$ has codimension at least 6 . Take for example the "bad condition" given by the vanishing of all 6 polynomials of (3.2).

This defines a map

$$
\mathbb{R}^{3} \times V \rightarrow \mathbb{R}^{6}
$$

and all we have to do (!) is to verify that the defining polynomials are irreducible and that this map has rank 6 at some point whose image is zero. Naturally we spare the reader the details of such verifications. Note that when $k=4$ we obtain a "bad condition" given by the vanishing of the first 4 polynomials of (3.2) and either

$$
a_{3}=b_{3}=c_{2}+d_{1} a_{2}-d_{3} x_{2}+e_{1} b_{2}-e_{3} x_{3}=0
$$

or

$$
a_{3}=b_{3}=a_{2} b_{4}-a_{4} b_{2}=0 \text {. }
$$


But this is not 7 conditions since the third condition of (3.2) becomes redundant. In this way we prove our final result.

(6.3) THEOREM. The isotopies, for which all the transitions on the focal set are of the four types in (3.4), are generic (open and dense).

\section{REFERENCES}

1. V. I. Arnold, Wavefront evolution and equivariant Morse lemma, Comm. Pure Appl. Math. 29 (1976), 557-582.

2. V. I. Arnold, Catastrophe theory (Springer-Verlag, 1984).

3. J. W. Bruce, Wavefronts and parallels in Euclidean space, Math. Proc. Cambridge Philos. Soc. 93 (1983), 323-333.

4. J. W. Bruce, Isotopies of generic plane curves Glasgow Math. J. 24 (1983), 195-206.

5. J. W. Bruce, Self-intersections of wavefront evolution, Proc. Roy. Irish Acad. Sect. A. 83 (1983), 225-229.

6. J. W. Bruce and P. J. Giblin, Curves and singularities (Cambridge University Press, 1984).

7. J. W. Bruce and P. J. Giblin, Outlines and their duals, Proc. London Math. Soc (3) 50 (1985), 552-570.

8. O. P. Shcherbak, Projectively dual space curves and Legendre singularities (Russian), Trudy Tbiliss. Univ. 232/233 (1982), 280-336.

Department of Mathematics

THE UNIVERSITY

NEWCASTLE-UPON-TYNE NE1 7RU

ENGLAND
Department of Pure Mathematics

THE UNIVERSITY

LIVERPOOL L69 3BX

ENGLAND 